\title{
Recombination Phenotypes of Escherichia coli greA Mutants
}

Anthony R Poteete

\begin{abstract}
Background: The elongation factor GreA binds to RNA polymerase and modulates transcriptional pausing. Some recent research suggests that the primary role of GreA may not be to regulate gene expression, but rather, to promote the progression of replication forks which collide with RNA polymerase, and which might otherwise collapse. Replication fork collapse is known to generate dsDNA breaks, which can be recombinogenic. It follows that GreA malfunction could have consequences affecting homologous recombination.

Results: Escherichia coli mutants bearing substitutions of the active site acidic residues of the transcription elongation factor GreA, D41N and E44K, were isolated as suppressors of growth inhibition by a toxic variant of the bacteriophage lambda Red-beta recombination protein. These mutants, as well as a D41A greA mutant and a greA deletion, were tested for proficiency in recombination events. The mutations were found to increase the efficiency of RecA-RecBCD-mediated and RecA-Red-mediated recombination, which are replication-independent, and to decrease the efficiency of replication-dependent Red-mediated recombination.

Conclusion: These observations provide new evidence for a role of GreA in resolving conflicts between replication and transcription.
\end{abstract}

\section{Background}

The GreA protein is an evolutionarily-conserved bacterial transcriptional factor, which interacts directly with RNA polymerase. Binding of GreA to paused and backtracked transcriptional complexes induces RNA polymerase to hydrolyze the last few nucleotide residues at the 3' end of the nascent transcript protruding beyond the enzyme's catalytic center, and resume transcription, in vitro [1] and in vivo $[2,3]$. The extensively characterized interaction between RNA polymerase and GreA leads most simply to the idea that GreA functions in transcriptional regulation. Consistent with this idea, $E$. coli lacking GreA protein exhibits altered levels of expression of some of its genes $[4,5]$, as well as of the transcript encoding bacteriophage $\lambda$ proteins which are synthesized late in infection [6]. However, the magnitude of the gene expression effects is small, and a clear understanding of GreA's biological function remains to be obtained. Some recent studies have provided

Correspondence: anthony.poteete@umassmed.edu

Department of Microbiology and Physiological Systems, University of

Massachusetts Medical School, Worcester, MA, USA evidence that GreA may have a role in resolving conflicts between replication and transcription $[7,8]$.

In this study, greA mutants are shown to have effects on homologous recombination which can be most readily explained by a replication fork-preserving function of wild type GreA. These mutants were isolated in a screen for host functions which affect the cell's interaction with the $\operatorname{Red} \beta$ recombination protein of bacteriophage $\lambda$.

In wild-type $E$. coli, homologous recombination depends primarily on the function of two proteins, the RecA synaptase and the RecBCD helicase/nuclease. For genetic engineering purposes, RecBCD is often replaced by the Red recombination system of bacteriophage $\lambda$ [9]. The Red system, consisting of the single-strand annealing protein $\operatorname{Red} \beta$ and the exonuclease $\lambda$ Exo, can substitute for RecBCD in promoting RecA-dependent homologous recombination $[10,11]$. In addition, however, Red confers new capabilities on the cell, including the ability of the cell's chromosome to recombine with short linear DNA species, even in the absence of RecA $[12,13]$. In this RecA-independent mode of recombination, Red apparently acts by targeting the replisome, 
leading to either strand assimilation or a template switch $[14,15]$.

\section{Results}

To probe interactions between the Red system and cellular proteins, $\operatorname{Red} \beta$ was fused at its $C$-terminus to the SPA affinity tag [16] on a medium copy number plasmid, under control of the lac repressor. $\operatorname{Red} \beta$-SPA was found to be toxic, inhibiting the growth of wild type E. coli when induced. This toxicity is surprising, because $\operatorname{Red} \beta$ itself is innocuous, and hundreds of non-toxic SPA-tagged E. coli proteins have been made and characterized [17]. One possible explanation is that the synthetic toxicity of Red $\beta$-SPA results from the delivery of the SPA tag to a critical site, possibly the normal site of $\operatorname{Red} \beta$ action in the cell. As a hypothetical example, if $\operatorname{Red} \beta$ normally binds to a replisome protein, it would necessarily have evolved to do so without interfering with replication. However, $\operatorname{Red} \beta$ with the 69 amino acid residue SPA tag appended might bind to its normal site and clash sterically with another component of the replisome. According to this reasoning, cellular mutants altered in their interaction with $\operatorname{Red} \beta$ could be selected as suppressors of Red $\beta$-SPA toxicity. Several such suppressor mutants were isolated. Initial mapping showed that three of the mutations mapped to the same area in the E. coli chromosome. Fine-structure mapping and sequencing revealed that these three mutants each encoded a GreA protein with a single amino acid substitution: D41N (isolated twice), or E44K. The affected residues are particularly significant in GreA's activity. Both participate in the coordination of $\mathrm{Mg}^{++}$ions in the catalytic core of RNA polymerase [18-20]. The greA$\mathrm{D} 41 \mathrm{~N}$ mutation was found not to affect the level of either $\operatorname{Red} \beta$-SPA or $\operatorname{Red} \beta$ as detected by western blot (not shown), ruling out the simplest explanation-that the greA mutants suppress the toxicity of Red $\beta$-SPA by preventing its synthesis.

To study the unexpected relationship between GreA and Red, E. coli strains were constructed bearing the greA D $41 \mathrm{~N}$ and E44K mutations, as well as a D41A mutation and a simple greA deletion, in a variety of genetic backgrounds. The strains were found to have a number of recombination-related phenotypes.

Consistent with the idea that the greA mutants might be affected in their interaction with Red, $\lambda$ behaves as if it had a mutation in either red gene (bet or exo), or both, when infecting these mutant bacteria. A $\lambda$ red gam double mutant does not form plaques on a recA host, though red and gam single mutants do so [21]. As indicated in Table 1 , a $\lambda$ gam single mutant fails to form plaques on a rec $A$ host when the $\operatorname{rec} A$ host additionally bears a gre $A$ point mutation (but not a gre $A$ deletion). A deletion eliminating both red genes reduces
Table $1 \lambda$ plaque formation ${ }^{a}$

\begin{tabular}{lccc}
\hline Host genotype $^{\mathbf{b}}$ & $\boldsymbol{\lambda}$ wild & $\boldsymbol{\lambda}$ gam & $\boldsymbol{\lambda}$ red \\
\hline wild type & ++ & + & + \\
\hline greA-D41N & + & - & + \\
\hline greA-D41A & + & - & + \\
\hline greA-E44K & + & - & + \\
\hline greA $\triangle$ & ++ & + & + \\
\hline greB $\triangle$ & ++ & + & + \\
\hline greB $\triangle$ greA-D41N & + & - & + \\
\hline
\end{tabular}

a. ++ large plaques, + small plaques, - no plaques.

b. All strains bear a recADtet substitution.

$\lambda$ plaque size; similarly, the greA point mutations in the host reduce the size of wild type $\lambda$ plaques. As might be expected if mutating either red or greA is equivalent in this test, the greA point mutations have less effect on the plaque size of a $\lambda$ red mutant than on the plaque size of $\lambda$ wild type. This latter observation suggests that the gre $A$ point mutation primarily affects $\lambda$ red function, rather than $\lambda$ transcription, but does not rule out the possibility that there might be some effect on transcription.

The effects of gre $A$ mutants on $\lambda$ recombination were tested more directly in a marker rescue test, shown in Figure 1. The crosses were done in a recA host, to restrict recombination to the Red-promoted, replisome invasion pathway [15]. Marker rescue was reduced 3-4 fold by the D41N and E44K substitutions, 2-fold by $\mathrm{D} 41 \mathrm{~A}$, and very slightly by the greA deletion. The effects of the greA mutations on Red-mediated recombination are modest compared to the effects of a red deletion mutant, which reduces plaque formation in this test to the background of reversion, approximately 40 -fold (data not shown).

The greA mutations also affect Red-mediated recombination outside the context of a phage infection. As shown in Figure 2, the efficiency of recombination between the bacterial chromosome and a linear dsDNA introduced by electroporation is reduced in the greA mutants, which exhibit the same rank order of effects as in the marker rescue experiment.

Recombination via the RecA-RecBCD pathway is also affected by greA mutations. P1 transduction of the Tn10 tet $R A$ genes inserted into several different locations in the chromosome was tested in the D $41 \mathrm{~N}$ mutant. In most, but not all cases, the mutant gave rise to more transductants than the wild type. A marker consisting of the $m e t B$ gene replaced by tetRA was typical. As shown in Figure 3, the efficiency of transduction of metBAtet is elevated by all the greA mutations. Again, the rank order of effects is the same, though in the opposite direction. The elevated frequency of transduction in the greA mutants could be attributed to an elevated 

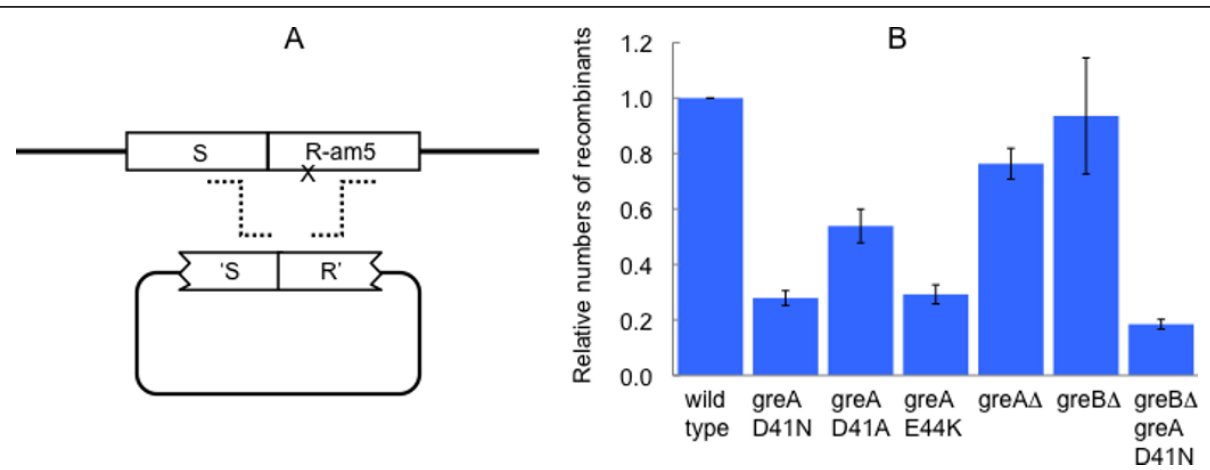

Figure 1 Marker rescue. A. $\lambda$ c1857 R-am5, bearing an amber mutation in the endolysin gene R, can form a plaque on the non-suppressing host only if it recombines with the plasmid or reverts. B. Efficiencies of mutants relative to wild type. Titers of the phage on cells bearing the homology-containing plasmid, minus titers on cells bearing the vector (revertants) are determined, and divided by the wild type value. Means and standard errors of four experiments are shown. All strains are recA.

frequency of recombination, rather than to a greater efficiency of P1 infection or tetRA expression, because P1 transduction of tetRA at some other loci, for example pro $A B$ or $r e c A$, was not elevated (data not shown).

To characterize further the specificity of greA recombination effects, the effect of the greA-D $41 \mathrm{~N}$ mutation on RecA-mediated recombination was tested in a strain in which $\operatorname{rec} B C D$ was replaced by red. As shown in Figure 3, the replacement has little effect, in either a GreA+ or a greA-D $41 \mathrm{~N}$ background. In particular, the greA mutation stimulates RecA-Red recombination at least as much as it stimulates RecA-RecBCD recombination.

The GreB protein of $E$. coli is very similar to GreA in structure and biochemical activities [22]. The transcriptional effects of a greA deletion mutant are often greater in a strain in which greB is deleted as well $[23,6,5,8]$. However, as shown in Table 1 and Figures 1, 2, and 3, deletion of greB had little or no effect on recombination, either by itself or in combination with greA-D $41 \mathrm{~N}$.

The DksA protein of E. coli, compared to GreB, is more distantly related to GreA, but it shares a number of structural and mechanistic features with the Gre proteins, including an acid-tipped coiled coil which reaches into the secondary channel of RNA polymerase to regulate transcription $[24,25]$. Chromosomal $d k s A$ mutants corresponding to the gre $A$ mutants described above were constructed and tested for recombination phenotypes: a deletion fusing the first three and last three codons, and a double substitution mutation neutralizing both of the acid fingertip residues, D71N/D74N [24,26]. In contrast to the gre $A$ mutants, neither $d k s A$ mutant exhibited either depressed or elevated levels of Redmediated or RecA-RecBCD-mediated recombination, nor was either resistant to Red $\beta$-SPA (data not shown).

The mechanism by which the greA point mutations suppress $\operatorname{Red} \beta$-SPA toxicity was investigated by means of a complementation test. Wild type, D41N, and E44K alleles of greA, under control of their own promoters, were cloned in multicopy plasmids which are compatible with the Red $\beta$-SPA expressing plasmids. The wild type greA plasmid was found to restore Red $\beta$-SPA sensitivity to a chromosomal greA-D $41 \mathrm{~N}$ mutant, indicating

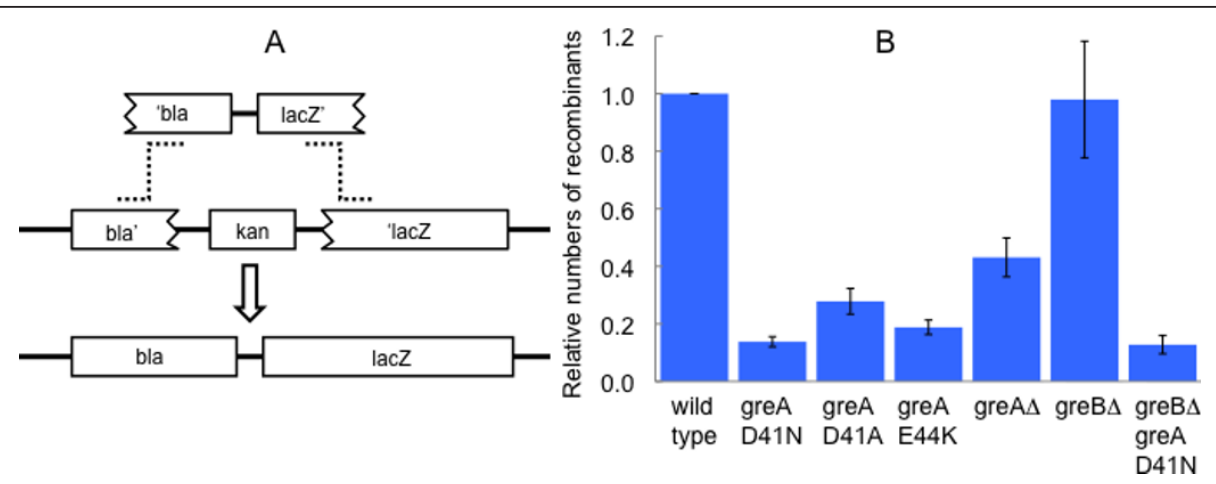

Figure 2 Red-mediated chromosomal gene replacement. A. Strains bearing an alteration of the lac locus in which lacl through the Nterminal coding region of lacZ is replaced by the C-terminal two-thirds of the bla gene and the aph gene of Tn903 can recombine with a linear dsDNA generated by PCR and introduced by electroporation, creating an ampicillin-resistant, Lac+ recombinant. B. Efficiencies of mutant strains relative to wild type. Means and standard errors of three or four experiments are shown. 

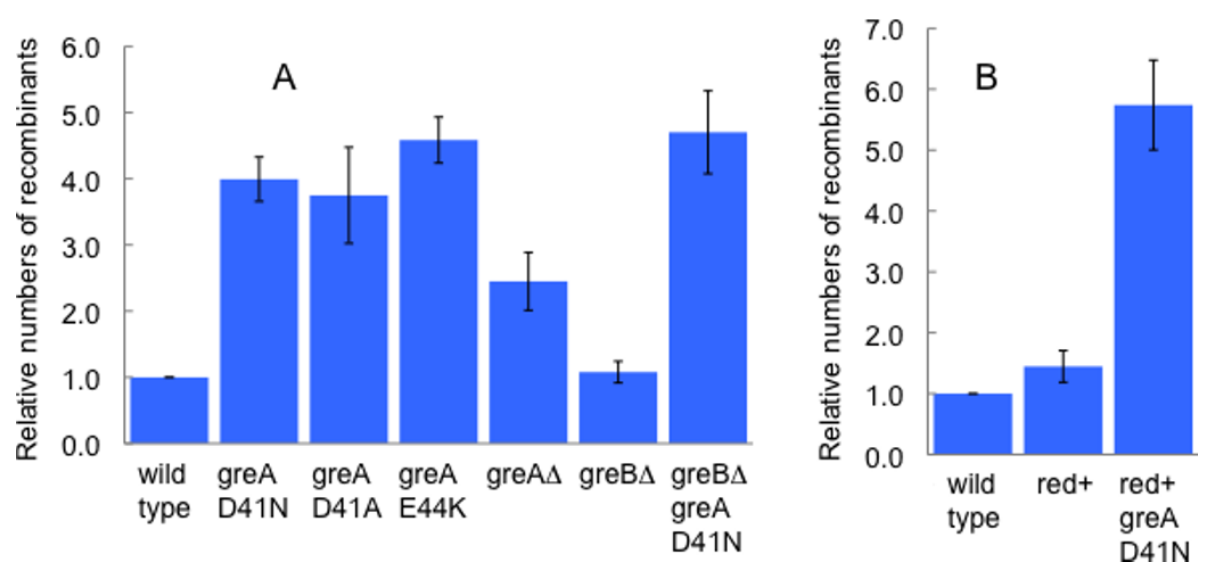

Figure 3 Transduction. Strains were infected with an amount of a P1 lysate grown on a metBAtet strain sufficient to generate 50-100 transductants in the wild type. Ratios of mutant transductants to wild type transductants were calculated. Means and standard errors of 3-8 crosses are shown. A. RecBCD+ strains. B. Strains in which recBCD is replaced by red.

that the suppression of $\operatorname{Red} \beta$-SPA toxicity does not result from a gain of function by the mutant GreA protein. Consistent with this conclusion, and more remarkably, the mutant plasmids do not confer $\operatorname{Red} \beta-\mathrm{SPA}$ resistance upon wild type $E$. coli, indicating that the wild type phenotype prevails even when the mutant gene has a higher copy number. However, interpretation of this latter result is complicated because the mutant plasmids significantly slow the growth of wild type cells, as expected based on the known toxicity of the mutant GreA proteins expressed at high levels [18].

The known biochemical properties of GreA substitution mutants suggest that one effect of the D41N or E44K mutation would be to increase the amount of time RNA polymerase spends stalled in certain backtracked transcriptional complexes. In the mutant, it is likely that other cellular proteins which respond to stalled transcriptional complexes will play a greater role in resolving them. One such protein is Mfd, which not only dissociates stalled transcription complexes, but at the same time recruits proteins that repair DNA lesions [27]. This line of reasoning leads to the question of whether mutant GreA suppression of $\operatorname{Red} \beta$-SPA toxicity is mediated by Mfd. Accordingly, wild type and greA$\mathrm{D} 41 \mathrm{~N}$ strains lacking the $m f d$ gene were constructed. Deletion of $m f d$ was found to have no effect on either the $\operatorname{Red} \beta$-SPA resistance of the D41N mutant, or the Red $\beta$-SPA sensitivity of wild type. It can be concluded that Mfd is not required for greA-D41N suppression of Red $\beta$-SPA toxicity.

\section{Discussion and Conclusions}

The experiments described here show that E. coli greA mutants exhibit elevated levels of RecA-mediated recombination, and depressed levels of Red-mediated,
RecA-independent recombination. GreA is a transcription factor, so these differing effects could be due to changes in gene expression, but recombination and DNA metabolism functions are not among the genes identified as significantly up- or down-regulated in a greA null mutant [5]. A more likely explanation comes from the idea that GreA functions to prevent stalling of replication forks that run into conflicts with transcription complexes $[7,8]$.

Replication in E.coli is highly processive, and maintains this processivity despite the presence on the template DNA of transcription complexes which have an intrinsic ability to stall replication forks [28]. Stalled replication forks are a potentially dangerous source of genetic instability, because they generate double-strand breaks through a process of RuvABC-mediated reversal and cleavage [29]. The dsDNA ends resulting from these breaks are recombinogenic [30], so loss of a function which promotes the progression of replication forks is predicted to stimulate RecA-RecBCD and RecA-Red recombination events, which proceed via mechanisms that are largely replication-independent [31]. Redmediated recombination in the absence of RecA, on the other hand, is itself strongly replication-dependent $[15,32]$, so loss of a function which promotes the progression of replication forks might be expected to diminish it.

The greA mutant phenotypes reported here are consistent with a model in which replication forks stall either more frequently or for a longer time in a cell in which GreA is inactive. Replication processivity in the mutant then becomes dependent upon other transcriptionreplication mediators. A functionally defective GreA protein, such as $\mathrm{D} 41 \mathrm{~N}$ or $\mathrm{E} 44 \mathrm{~K}$, which can still bind to its normal site on RNA polymerase, might have an 
exacerbated effect, by competing with one or more of the other mediators.

Two recent studies present evidence for a role of GreA in promoting the progression of replication forks. (1) Deletion of greA increases the UV sensitivity of a ruvC mutant. This effect of the greA mutant is suppressed by the RNA polymerase open complexdestabilizing rpoB mutant $\mathrm{H} 1244 \mathrm{Q}$. The investigators also found that GreA and DksA together contribute to the viability of a $\operatorname{ruv} C$ strain. They interpreted their observations as indicating that these transcription factors act at DNA lesions to resolve conflicts between DNA replication and transcription [7]. (2) Cultures of an $E$. coli dnaC-ts mutant were synchronized by temperature shift, then, after replication initiation, treated with serine hydroxamate to induce amino acid starvation. The treated cells completed the round of replication they had started, but failed to do so if they lacked DksA, or if they lacked both GreA and GreB. The replication block could be eliminated by additional treatment with rifampicin, or by a DksA-bypassing mutation in rpoB, $\mathrm{P} 546 \mathrm{~L}$, indicating that RNA polymerase was responsible for the replication block, and that the transcription factors affected replication through their wellcharacterized interaction with RNA polymerase. The replication deficiency of the $d k s A$ mutant in this experiment could be complemented by overexpression of GreA from a plasmid. In synchronized dnaC mutant cells that were not treated with serine hydroxamate, deletion of $d k s A$ slightly reduced the rate of replication fork progression; deletion of $d k s A$, gre $A$, and gre $B$ all together substantially reduced it. [8]. To these two lines of evidence, a third is now added: in otherwise wild type cells, not treated with DNA damaging agents or metabolic inhibitors, greA mutations stimulate replication-independent recombination, and depress replication-dependent recombination.

Stimulation of replication-independent, RecARecBCD-mediated recombination was not uniform, but rather was found to vary among different loci. This observation is consistent with the idea that the stimulation is due to the production of dsDNA ends as a byproduct of replication-transcription conflicts: such conflicts would not be expected to occur at a uniform frequency around the chromosome, because different loci are transcribed at different rates.

Alteration of either of GreA's key active site residues has stronger recombination effects than simply eliminating GreA. This observation is consistent with previous studies of many GreA mutants, including D41N and D41A as well as other substitutions for both D41 and E44. These mutants generally reduce or eliminate GreAinduced transcript cleavage, but not binding to RNA polymerase [18-20]. Unlike wild type GreA, the mutant proteins inhibit elongation when used at high concentration in vitro [18]. One unexplained observation of the present study is that the D41A substitution was found to have smaller effects on recombination than the $\mathrm{D} 41 \mathrm{~N}$ substitution, whereas Laptenko et al. [18] found little if any difference in biochemical activities between these two mutants.

Wang and coworkers propose that the various transcription-replication mediators, including DksA, GreA, GreB and Mfd, could be specialized to prevent transcription-replication conflict under different conditions [8]. The experiments described here provide additional support for this interpretation: based on the recombination and Red $\beta$-SPA sensitivity phenotypes, among these proteins, only GreA appears to have a significant role in mediating transcription-replication conflicts that arise during rapid growth in rich medium. Some qualitative observations on the growth properties of greA and $d k s A$ mutants are also consistent with this idea. The gre $A$ point mutants grow noticeably more slowly than either wild type or the greA deletion on rich medium (LB), but about as well as wild type on minimal medium (M9 glucose-thiamine). In contrast, the $d k s A$ D 71N/D74N mutant grows about as well as wild type on LB, but only a little better on M9 glucose-thiamine than the $d k s A$ deletion mutant, which, as previously reported, grows very poorly on minimal medium [33].

\section{Methods}

\section{Strain construction}

$\operatorname{Red} \beta$ was fused to the SPA affinity tag as described by Zeghouf et al. [16], on plasmid pKM208 [34]. The resulting plasmid, pTP1205, contains the lacI gene, a synthetic operon in which $\lambda$ gam and $\operatorname{red} \beta-S P A$ are transcribed from Ptac, and genes conferring resistance to ampicillin (bla) and kanamycin (neo). A derivative, pTP1206, was made by deleting bla. Further plasmid construction details are given in Table 2 . The growth of wild type $E$. coli bearing pTP1205 or pTP1206, but not pKM208, is inhibited by addition of the inducer isopropyl- $\beta$-D-thiogalactoside (IPTG). For selection of $\operatorname{Red} \beta$ SPA-resistant mutants, the reduced-genome $E$. coli strain MDS12 [35] was mutagenized with $N$-methyl- $N$ 'nitro- $N$-nitrosoguanidine plus chloramphenicol [36]. Plasmid pTP1206 was introduced into the mutagenized cells by electroporation. Kanamycin- and IPTG-resistant colonies were selected, and then screened, or reselected, for the ability to grow while bearing pTP1205 in the presence of IPTG.

Mapping of the Red $\beta$-SPA-resistant mutants was done as described by Ortenberg et al. [37], except that the mini transposon conferred resistance to tetracycline instead of chloramphenicol. Each mutant was infected with $\lambda$ NK1098 to generate a population with random 
Table 2 Plasmids

\begin{tabular}{|c|c|}
\hline$\overline{\mathrm{pCP} 20}$ & [45]. Temperature-sensitive, with heat-inducible FLP \\
\hline pKM208 & $\begin{array}{l}\text { [34]. bla-Ptac-gam-bet-exo-lacl, temperature-sensitive } \\
\text { mutant pSC101 origin }\end{array}$ \\
\hline pJL148 & [16]. SPA-neo \\
\hline pTP1068 & $\begin{array}{l}\text { Plasmid containing bla- } P_{32} \text {-aacC. See the description of } \\
\text { pTP1232 below. }\end{array}$ \\
\hline pTP1205 & $\begin{array}{l}\text { pKM } 208 \times \text { SP01,02 pcr of pJL148. The Red-mediated } \\
\text { recombination event fuses the SPA tag to the C- } \\
\text { terminal end of bet, deletes most of exo, and adds } \\
\text { neo. }\end{array}$ \\
\hline pTP1206 & $\begin{array}{l}\text { Deletion of bla from pTP1205 by digestion with Pvul } \\
\text { and religation }\end{array}$ \\
\hline pTP1214 & $\begin{array}{l}\text { pKM } 208 \times \text { cat } 29,30 \text { pcr of Tn9 cat. The Red-mediated } \\
\text { recombination event replaces a segment of pKM } 208 \\
\text { between lacl and the replication origin with the cat } \\
\text { gene, flanked by BamH1 sites. }\end{array}$ \\
\hline pTP1215 & $\begin{array}{l}\text { Deletion of cat from pTP1214 by digestion with } \\
\text { BamH1 and religation }\end{array}$ \\
\hline pTP1216 & $\begin{array}{l}\text { pTP1215 } \times \text { cat } 27,28 \text { pcr of Tn9 cat. The Red-mediated } \\
\text { recombination event replaces bla and the f1 } \\
\text { replication origin with cat. Smaller, chloramphenicol } \\
\text { resistance-conferring version of pKM208. }\end{array}$ \\
\hline pTP1222 & $\begin{array}{l}\text { A segment of } \lambda \text { wild type DNA was amplified by SR2,3 } \\
\text { pcr. The product was digested with BamH1 and Xbal, } \\
\text { and ligated with a BamH1- and Xbal-ended plasmid } \\
\text { segment consisting of the Tn903 aph gene and the } \\
\text { pSC101 replication origin. }\end{array}$ \\
\hline pTP1223 & $\begin{array}{l}\text { Deletion of sequences between the EcoRV and Xbal } \\
\text { sites of pTP1222. The resulting plasmid contains } \lambda \\
\text { sequences from } 45262 \text { in the S gene to } 45828 \text { in the R } \\
\text { gene. }\end{array}$ \\
\hline $\begin{array}{l}\text { pTP1228, 1230, } \\
1231\end{array}$ & $\begin{array}{l}\text { Wild type, D41N, and E44K alleles of greA, respectively, } \\
\text { amplified by PCR with primers greA5 and greA6, and } \\
\text { cloned between the EcoR1 and BamH1 sites of a } \\
\text { derivative of pBR322 lacking the sequences between } \\
\text { the BamH1 and Pvull sites. }\end{array}$ \\
\hline pTP1232 & $\begin{array}{l}\text { Contains the sequences used to construct TP1234, } \\
\text { DNA from which in turn was used as the template for } \\
\text { PCR synthesis of the 587-bp dsDNA used in the tests } \\
\text { of Red-mediated chromosomal gene replacement } \\
\text { (sequence shown in Figure 4). It includes the bla gene } \\
\text { of pBR322, the synthetic, unregulated promoter P } 32 \\
\text { (closely related to CP32 [46]), the lacZ ribosome } \\
\text { binding site, and the N-terminal end of lacZ. }\end{array}$ \\
\hline
\end{tabular}

tetracycline-resistant transposon insertions. Lysates of phage P1 grown on the populations were used to transduce wild type cells to tetracycline- and $\operatorname{Red} \beta$ SPA-resistance simultaneously. The mutation-linked transposons were then located by the use of arbitrary PCR and sequencing; oligonucleotides used for this purpose are described in Table 3. The three greA mutants were first localized to the vicinity of $y r b D$ and $y r b C$. An $\operatorname{argG}:: c a t$ insertion was then constructed, and three-factor crosses were employed to determine that the location of the mutations was between $\operatorname{argG}$ and $y r b D$ (91\% and 86\% co-transduction, respectively). Sequencing of the region revealed that the three mutants had base substitutions altering greA codons 41 (GAC to AAC, D41N, isolated twice independently) or 44 (GAA to AAA, E44K), among other, uncorrelated mutations in nearby genes.

Mutations were introduced into the greA gene of E. coli strain MG1655 by the use of Red-mediated recombination, in a two-step process. The first two bases of codon 41 or 44 were replaced with a tetracyclineresistance cassette. Oligonucleotides were then used to delete the cassette and insert the new bases [9]. Recombinants were identified following kanamycin enrichment for tetracycline-sensitives; details of the protocol are given below. A deletion allele fusing the first three and last three codons of greA was constructed the same way. The greA alleles were verified by sequencing. The same processes were used to construct chromsomal $d k s A-D 71 \mathrm{~N} / \mathrm{D} 74 \mathrm{~K}$ and $d k s A$ deletion mutants, except that the initial construction replaced ten bases in codons 71-74 with the cat gene. The greB mutation used in some of the experiments was introduced by P1 transduction as greB $\Delta$ kan-FRT; the kanamycin resistance element in it was subsequently excised by the use of FLP recombinase as described [38]. The $m f d$ gene was deleted the same way. Other genetic markers were introduced into greA, greB, or $d k s A$ mutant strains by P1 transduction. Further strain construction details are given in Table 4.

\section{Kanamycin enrichment for chloramphenicol-sensitive or tetracycline-sensitive variants}

Following electroporation, bacteria were diluted into $15 \mathrm{ml}$ of $\mathrm{LB}$, and left standing overnight at $30^{\circ} \mathrm{C}$ for complete recovery from electroporation and outgrowth of fully sensitive recombinants. $0.2 \mathrm{ml}$ of the culture was diluted into $5 \mathrm{ml} \mathrm{LB}$ and aerated at $37^{\circ} \mathrm{C}$ until a density of about $7 \times 10^{7} / \mathrm{ml}$ was reached. At that point, $2.5 \mathrm{ml}$ of culture was mixed with $2.5 \mathrm{ml} \mathrm{LB}$ plus $100 \mu \mathrm{l}$ of either chloramphenicol at $1 \mathrm{mg} / \mathrm{ml}$ or tetracycline at $0.4 \mathrm{mg} / \mathrm{ml}$, and aerated at $37^{\circ} \mathrm{C}$ for $40 \mathrm{~min}$. At that time, $2 \mathrm{ml}$ of culture was mixed with $2 \mathrm{ml} \mathrm{LB}$ plus $40 \mu \mathrm{l}$ chloramphenicol or tetracycline, plus $4 \mu \mathrm{l}$ kanamycin at $20 \mathrm{mg} / \mathrm{ml}$ (for a final kanamycin concentration of $20 \mu \mathrm{g} / \mathrm{ml}$ ), and aerated at $37^{\circ} \mathrm{C}$ for $40 \mathrm{~min}$, then left standing overnight at room temperature. Portions of the culture were plated on LB, and the resulting colonies were tested for chloramphenicol or tetracycline sensitivity. Clones bearing the intended deletion were identified by the use of PCR and sequencing.

\section{Phages}

$\lambda$ NK1098 bears the mutations cI857 and P-am80, as well as a mini Tn10 tet element and the Tn10 transposase fused to Ptac. It is similar to $\lambda$ NK1323 [39], but its transposase is wild type, rather than the Altered Target Specificity double mutant version borne by $\lambda$ NK1323. The amber mutation R5 [40], used in marker rescue 
Table 3 Oligos

\begin{tabular}{|c|c|}
\hline aof2 & TCCGTAATCATGGTCATAGCTGTTTCCTGTGTGAAATTGTTTAGGTGGCGGTACTTGGGT \\
\hline $\mathrm{ARB1}^{\mathrm{a}}$ & GGCCACGCGTCGACTAGTACNNNNNNNNNNGATAT \\
\hline$\overline{\mathrm{ARB}^{\mathrm{a}}}$ & GGCCACGCGTCGACTAGTACNNNNNNNNNNACGCC \\
\hline $\mathrm{ARB6}^{\mathrm{a}}$ & GGCCACGCGTCGACTAGTAC \\
\hline $\mathrm{bpa}$ & TCTGGTGGCCGGAAGGCGAAGCGGCATGCATTACGTTGAGGTCTGACAGTTACCAATGC \\
\hline BLZ3 & GAACTTTAAAAGTGCTCATCATTG \\
\hline BLZ4 & GGGTITTCCCAGTCACG \\
\hline cat27 & TCGGAGATCCCCCGGGCTGCAGGAATTCGATATCAAGCTTATGAGACGTTGATCGGCACG \\
\hline cat28 & AGTGGAACGAAAACTCACGTTAAGGGATITTGGTATGAGATTCAGGCGTAGCACCAGGC \\
\hline cat29 & GACAGGTTTCCCGACTGGAAAGCGGGCAGTGAGCGCAACGGATCCATGAGACGTTGATCGGCACG \\
\hline cat30 & TGGCTAAATACGGAAGGATCTGAGGTTCTTATGGCTCTTGGATCCATTCAGGCGTAGCACCAGGC \\
\hline cat34 & TCAAAATCGTCAACCCGATTATGGGCGTGAAATTCTGGGAATGAGACGTTGATCGGCACG \\
\hline cat35 & GCGTACTGTGACTTCTTCTGCCGGGATCTTCACGCTCTCAATTCAGGCGTAGCACCAGGC \\
\hline cat38 & AGGTTGATATTGATTCAGAGGTATAAAACGAATGAGTACTATGAGACGTTGATCGGCACG \\
\hline cat39 & CCTACCCGGATATTATCGTGAGGATGCGTCATCGCCATTGATTCAGGCGTAGCACCAGGC \\
\hline cat40 & CACCGTTACACATATGCAGGATGAAGCAGCCAACTTCCCGATGAGACGTTGATCGGCACG \\
\hline cat41 & CGCAGTTCGAGGCTGAACTCTTCTTCCTGGGCTGCACGGTATTCAGGCGTAGCACCAGGC \\
\hline D41A & AGCTGCGTGGTATTCGGCGTTTCTTTCAGGGCGCCATGCTCACGCGCTTCCGCGATAGCAGCAA \\
\hline $\mathrm{D} 41 \mathrm{~N}$ & AGCTGCGTGGTATTCGGCGTTTCTTTCAGGTTGCCATGCTCACGCGCTTCCGCGATAGCAGCAA \\
\hline $\mathrm{dksAD}$ & AGTGCGTGTTAAGGAGAAGCAACATGCAAGAAATGGCTGGCTAATTACAGCCGTTCCATCACGT \\
\hline $\mathrm{dksNN}$ & CATATGCAGGATGAAGCAGCCAACTTCCCGAACCCGGTAAACCGTGCAGCCCAGGAAGAAGAGTTCAGCC \\
\hline E44K & CTGTTCACGAGCTGCGTGGTATTCGGCGTTITITTCAGGTCGCCATGCTCACGCGCTTCCGCGA \\
\hline greA5 & CATCATCATGAATTCAGCTGGTCAATCCGATGA \\
\hline greA6 & ACTACTACTGGATCCTGTACAGGTCATCGGTAAAACG \\
\hline greA9 & TGAAATCATTGCTGCTATCGCGGAAGCGCGTGAGCATGGCCTCGACATCTTGGTTACCGT \\
\hline greAD & ACATCTTGAGTATTGGGTAATTCTTACAGGTATTCAGCTTGCATTTGTAATACCTCTTGAATATTCCTGA \\
\hline greAX & CTGTTCACGAGCTGCGTGGTATTCGGCGTTTTCTTCAGGCGCGGAATAACATCATTTGG \\
\hline greAY & GCTGCTATCGCGGAAGCGCGTGAGCATGGCGACCTGAAAGCTCGACATCTTGGTTACCGT \\
\hline greAZ & AGAAACCCTGCTGTTCACGAGCTGCGTGGTATTCGGCGTTCGCGGAATAACATCATTTGG \\
\hline PRO1 & TGCTCATCATTGGAAAACGTTCTTCGGGGCGAAAACTCTACAACCAATTAACCAATTCTG \\
\hline $\mathrm{PRO} 2$ & GTCACGACGTTGTAAAACGACGGCCAGTGAATCCGTAATCACGTTGTGTCTCAAAATCTC \\
\hline 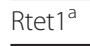 & CCACTTAATGTGATCATTGAACC \\
\hline${\text { Rtet } 2^{a}}^{a}$ & AACCATTITCAGTGATCCATTGC \\
\hline SP01 & ATTCCTGAAACAGAAAGCCGCAGAGCAGAAGGTGGCAGCATCCATGGAAAAGAGAAG \\
\hline SP02 & TTGCTCCCCAAATACAAAACCAATTTCAGCCAGTGCCTCGCATATGAATATCCTCCTTAG \\
\hline SR2 & GACTACGACTACATCTAGATTCGAACATCGATATGGGCAACTCTATC \\
\hline SR3 & ATCAGCCATCATGGATCCGTTTGCAATGGCGTACCTT \\
\hline
\end{tabular}

a. Arbitrary PCR and sequencing to locate transposon insertions was done as described [47], but with primers designed for Tn10d(tet). The first round of PCR was done with primers Rtet1, ARB1, and ARB6; the second with Rtet2 and ARB2. Sequencing was done with Rtet2.

experiments, was sequenced and found to change $\lambda$ 45710 from G-C to A-T, which turns R codon 73, UGG, into UAG. The $\gamma 210$ mutation [21], used in plating tests, was sequenced and found to change $\lambda 33076$ from G-C to A-T, which turns gam codon 53, CAG, into UAG. The deletion-substitution mutation red $\Delta c a t$, used in plating tests, was constructed by the use of Redmediated recombination [41], with a dsDNA made by PCR amplification of Tn9 cat with oligo primers cat38 and cat39 (Table 3). The deletion-substitution leaves in place the first three codons of bet and the last three codons of exo.

\section{Gene-replacement recombination test}

Strains to be tested for Red-mediated chromosomal gene replacement were transformed with plasmid pTP1216, a derivative of pKM208 in which bla is replaced by cat. They were grown at $30^{\circ} \mathrm{C}$ in $\mathrm{LB}$ broth 


\section{Table 4 Strains}

\begin{tabular}{|c|c|c|}
\hline MG1655 & Wild type & [43] \\
\hline MDS12 & Reduced genome version of MG1655 & [35] \\
\hline JW1100 & $\mathrm{mfd} \triangle \mathrm{kanFRT}$ & [38] \\
\hline JW3369 & greB $\triangle$ kanFRT & [38] \\
\hline TP796 & MDS12 recA $\triangle$ tet & [44] \\
\hline TP798 & MG1655 recBCD $\triangle$ Ptac-gam-bet-exo-cat & [44] \\
\hline \multicolumn{3}{|c|}{ Strains constructed for this study. All are in the MG1655 background } \\
\hline TP933 & recBCD $\triangle$ Ptac-gam-bet-exo-cat lacIP $\triangle:$ :bla-P 32 -aacC-lacZ & TP798 $\times$ bpa1,aof2 pcr of pTP1068 \\
\hline TP965 & recBCD $\triangle$ Ptac-gam-bet-exo-cat laclP $\triangle$ bla'-kan-'lacZ & TP933 $\times$ PRO1,2 pcr of Tn903 aph \\
\hline TP1195 & greA::tet41 & MG1655/pKM208 $\times$ greA9,X pcr of Tn10 tet \\
\hline TP1198 & greA-D41N & TP1195/pKM208 × oligo D41N \\
\hline TP1201 & recA $\triangle$ tet & MG1655 × P1·TP796 \\
\hline TP1202 & greA-D41N recA $\triangle$ tet & TP1198 × P1•TP796 \\
\hline TP1203 & greA:.tet44 & MG1655/pKM208 $\times$ greAY,Z pcr of Tn10 \\
\hline TP1204 & greA-D41A & TP1195/pKM208 × oligo D41A \\
\hline TP1205 & greA $\triangle$ & TP1195/pKM208 × oligo greAD \\
\hline TP1208 & greA-D41A recA $\Delta$ tet & TP1204 × P1•TP796 \\
\hline TP1209 & greA $\triangle$ recA $\triangle$ tet & TP1205 × P1·TP796 \\
\hline TP1213 & greA $\triangle$ greB $\Delta$ tet & TP1205 × P1·TP1199 \\
\hline TP1216 & greA-E44K & TP1203/pKM208 × oligo E44K \\
\hline TP1218 & greB $\triangle$ kanFRT & MG1655 × P1·JW3369 \\
\hline TP1219 & greA-E44K recA $\triangle$ tet & TP1216 × P1•TP796 \\
\hline TP1220 & greA-E44K greB $\triangle$ tet & TP1216 × P1•TP1199 \\
\hline TP1222 & greB $\triangle F R T$ & TP1218/pCP20 passaged at $42^{\circ}$ \\
\hline TP1224 & greA-D41N greB $\triangle$ kanFRT & TP1198 × P1•JW3369 \\
\hline TP1225 & laclP $\triangle$ bla'-kan-'lacZ & MG1655 × P1·TP965 \\
\hline TP1226 & greA-D41N laclP $\triangle$ bla'-kan-'lacZ & TP1198 × P1·TP965 \\
\hline$\overline{\mathrm{TP} 1227}$ & greA-D41A lacIP $\triangle$ bla'-kan-'lacZ & TP1204 × P1·TP965 \\
\hline TP1229 & greA-E44K lacIP $\triangle$ bla'-kan-'lacZ & TP1216 × P1·TP965 \\
\hline TP1230 & recBCD $\triangle$ red-cat greA-D41N & TP1198 × P1•TP798 \\
\hline TP1231 & greA-D41N greB $\triangle F R T$ & TP1224/pCP20 passaged at $41^{\circ}$ \\
\hline TP1232 & greB $\triangle F R T$ lacIP $\triangle$ bla'-kan-'lacZ & TP1222 × P1•TP965 \\
\hline TP1233 & greA-D41N greB $\triangle F R T$ laclP $\triangle$ bla'-kan-'lacZ & TP1231 × P1·TP965 \\
\hline TP1234 & laclP $\triangle:$ :bla-P 32 & TP1225/pTP1216 × pTP1232 linear \\
\hline TP1235 & greB $\triangle F R T$ recA $\triangle$ tet & TP1222 × P1•TP796 \\
\hline TP1236 & greA-D41N greB $\triangle F R T$ recA $\triangle$ tet & TP1231 × P1•TP796 \\
\hline TP1241 & greA $\triangle$ cat laclP $\triangle$ bla'-kan-'lacZ & TP1193 × P1•TP965 \\
\hline TP1242 & greA $\triangle$ lacIP $\triangle$ bla'-kan-'lacZ & TP1205 × P1•TP965 \\
\hline TP1252 & $\mathrm{mfd} \Delta \mathrm{kanFRT}$ & MG1655 × P1·JW1100 \\
\hline TP1253 & greA-D41N mfd $\triangle$ kanFRT & TP1198 × P1.JW1100 \\
\hline TP1254 & greA-E44K mfd $\triangle$ kanFRT & TP1216 × P1.JW1100 \\
\hline TP1255 & $\mathrm{dks} A \triangle 10:$ :cat & MG1655/pKM208 $\times$ cat40,41 pcr of Tn9 \\
\hline TP1257 & $\mathrm{mfd} \triangle \mathrm{FRT}$ & TP1252/pCP20 passaged at $41^{\circ}$ \\
\hline TP1258 & greA-D41N mfd $\triangle F R T$ & TP1253/pCP20 passaged at $41^{\circ}$ \\
\hline TP1259 & greA-E44K mfd $\triangle F R T$ & TP1254/pCP20 passaged at $41^{\circ}$ \\
\hline TP1260 & dksA-D71N/D74N & TP1255/pKM208 × oligo dksNN \\
\hline TP1262 & dksA-D71N/D74N recA $\Delta$ tet & TP1262 × P1·TP796 \\
\hline TP1263 & $\mathrm{dksA} \triangle$ & TP1255/pKM208 × oligo dksAD \\
\hline TP1264 & $\mathrm{dks} A \Delta \mathrm{rec} A \Delta \mathrm{tet}$ & TP1263 × P1.TP796 \\
\hline
\end{tabular}




\section{GAACTTTAAAAGTGCTCATCATTGGAAAACGTTCTTCGGGGCGAAA ACTCTCAAGGATCTTACCGCTGTTGAGATCCAGTTCGATGTAACCC ACTCGTGCACCCAACTGATCTTCAGCATCTTTTACTTTCACCAGCG TTTCTGGGTGAGCAAAAACAGGAAGGCAAAATGCCGCAAAAAAGGG AATAAGGGCGACACGGAAATGTTGAATACTCATACTCTTCCTTTTT CAATATTATTGAAGCATTTATCAGGGTTATTGTCTCATGAGCGGAT ACATATTTGAATGTATTTAGAAAAATAAACAAATAGGGGTTCCGCG CACATTTCCCCGAAAAGTGCCACCTGACGTCTAAGAAACCATTATT ATCATGACATTAACCTATAAAAATAGGCGTATCACGAGGCCCTTTC GTCTTCAAGAATTGCCGGCGGATCCATACGGGAGTTTATTCTGACA TATTGCCGGTGTGTTGGTATAATAACTTAGTAAGCTAATTCTAGCA CAATTTCACACAGGAAACAGCTATGACCATGATTACGGATTCACTG GCCGTCGTTTTACAACGTCGTGACTGGGAAAACCC}

Figure 4 Sequence of the 587-bp dsDNA used in the tests of Red-mediated chromosomal gene replacement. The first and last 50 bases are the homology flanks. Bases in bold type are, in order, the start codon of bla (reverse complement), the -35 and -10 hexamers of $\mathrm{P}_{32}$, and lac $\mathrm{Z}$ ribosome binding site.

supplemented with chloramphenicol at $20 \mu \mathrm{g} / \mathrm{ml}$, induced with $1 \mathrm{mM}$ IPTG, and prepared for electroporation as described by Murphy and Campellone [34]. Cells were electroporated with a mixture of $150 \mathrm{ng}$ of a dsDNA generated by PCR of plasmid pTP1232 (Table 2) with oligo primers BLZ3 and BLZ4 (Table 3), plus $1 \mathrm{ng}$ of plasmid pMB9 [42]. The linear dsDNA was $587 \mathrm{bp}$, including $50 \mathrm{bp}$ homology-targeting flanks (Figure 4). Following electroporation, the cells were diluted into $3 \mathrm{ml} \mathrm{LB}$ broth, grown with aeration for $2 \mathrm{hr}$ at $37^{\circ} \mathrm{C}$, and plated at $37^{\circ} \mathrm{C}$ on LB agar supplemented with ampicillin at $100 \mu \mathrm{g} / \mathrm{ml}$, or tetracycline at $15 \mu \mathrm{g} / \mathrm{ml}$. To adjust for variable growth rates and electroporation efficiencies, the ratio of ampicillin-resistant (recombinant) to tetracycline-resistant (transformant) colonies, normalized to that of the wild type control, was used as a measure of relative recombination proficiency.

\section{Acknowledgements}

I thank Hieuhanh Nguyen, Emily Rittershaus, and Mylorde Cherenfant for technical assistance; Martin Marinus and Jack Greenblatt for strains; and Sergei Borukhov and Jade Wang for helpful discussions.

Received: 19 December 2010 Accepted: 31 March 2011 Published: 31 March 2011

\section{References}

1. Borukhov S, Polyakov A, Nikiforov V, Goldfarb A: GreA protein: a transcription elongation factor from Escherichia coli. Proc Natl Acad Sci USA 1992, 89(19):8899-8902.

2. Toulme F, Mosrin-Huaman C, Sparkowski J, Das A, Leng M, Rahmouni AR: GreA and GreB proteins revive backtracked RNA polymerase in vivo by promoting transcript trimming. EMBO J 2000, 19(24):6853-6859.

3. Hatoum A, Roberts J: Prevalence of RNA polymerase stalling at Escherichia coli promoters after open complex formation. Mol Microbiol 2008, 68(1):17-28.

4. Susa $\mathrm{M}$, Kubori $\mathrm{T}$, Shimamoto $\mathrm{N}$ : A pathway branching in transcription initiation in Escherichia coli. Mol Microbiol 2006, 59(6):1807-1817.

5. Stepanova E, Lee J, Ozerova M, Semenova E, Datsenko K, Wanner BL, Severinov K, Borukhov S: Analysis of promoter targets for Escherichia coli transcription elongation factor GreA in vivo and in vitro. J Bacteriol 2007, 189(24):8772-8785
6. Marr MT, Roberts JW: Function of transcription cleavage factors GreA and GreB at a regulatory pause site. Mol Cell 2000, 6(6):1275-1285.

7. Trautinger BW, Jaktaji RP, Rusakova E, Lloyd RG: RNA polymerase modulators and DNA repair activities resolve conflicts between DNA replication and transcription. Mol Cell 2005, 19(2):247-258.

8. Tehranchi AK, Blankschien MD, Zhang Y, Halliday JA, Srivatsan A, Peng J, Herman C, Wang JD: The transcription factor DksA prevents conflicts between DNA replication and transcription machinery. Cell 2010, 141(4):595-605.

9. Court DL, Sawitzke JA, Thomason LC: Genetic engineering using homologous recombination. Annu Rev Genet 2002, 36:361-388.

10. Weisberg RA, Sternberg N: Transduction of recB-hosts is promoted by $\lambda$ red+ function. In Mechanisms in recombination. Edited by: Grell RF. pubNew York: Plenum Publishing Corp; 1974:107-109.

11. Poteete AR, Volkert MR: Activation of recF-dependent recombination in Escherichia coli by bacteriophage $\lambda$ and P22-encoded functions. J Bacteriol 1988, 170:4379-4381.

12. Murphy KC: Use of bacteriophage $\lambda$ recombination functions to promote gene replacement in Escherichia coli. J Bacteriol 1998, 180:2063-2071.

13. Yu D, Ellis HM, Lee EC, Jenkins NA, Copeland NG, Court DL: An efficient recombination system for chromosome engineering in Escherichia coli. Proc Natl Acad Sci USA 2000, 97:5978-5983.

14. Ellis HM, Yu D, DiTizio T, Court DL: High efficiency mutagenesis, repair, and engineering of chromosomal DNA using singlestranded oligonucleotides. Proc Natl Acad Sci USA 2001, 98(12):6742-6746.

15. Poteete AR: Involvement of DNA replication in phage lambda Redmediated homologous recombination. Mol Microbiol 2008, 68(1):66-74.

16. Zeghouf M, Li J, Butland G, Borkowska A, Canadien V, Richards D, Beattie B, Emili A, Greenblatt JF: Sequential Peptide Affinity (SPA) system for the identification of mammalian and bacterial protein complexes. J Proteome Res 2004, 3(3):463-468.

17. Butland G, Peregrin-Alvarez JM, Li J, Yang W, Yang X, Canadien V, Starostine A, Richards D, Beattie B, Krogan N, et al: Interaction network containing conserved and essential protein complexes in Escherichia coli. Nature 2005, 433(7025):531-537.

18. Laptenko O, Lee J, Lomakin I, Borukhov S: Transcript cleavage factors GreA and GreB act as transient catalytic components of RNA polymerase. EMBO J 2003, 22(23):6322-6334

19. Opalka N, Chlenov M, Chacon P, Rice WJ, Wriggers W, Darst SA: Structure and function of the transcription elongation factor GreB bound to bacterial RNA polymerase. Cell 2003, 114(3):335-345.

20. Sosunova E, Sosunov V, Kozlov M, Nikiforov V, Goldfarb A, Mustaev A Donation of catalytic residues to RNA polymerase active center by transcription factor Gre. Proc Natl Acad Sci USA 2003, 100(26):15469-15474.

21. Zissler J, Singer E, Schaefer F: The role of recombination in the growth of bacteroiophage $\lambda$. I. The Gamma gene. In The bacteriophage lambda. Edited by: Hershey AD. Cold Spring Harbor, N.Y.: Cold Spring Harbor Laboratory; 1971:455-468.

22. Borukhov S, Sagitov V, Goldfarb A: Transcript cleavage factors from E. coli. Cell 1993, 72(3):459-466.

23. Orlova M, Newlands J, Das A, Goldfarb A, Borukhov S: Intrinsic transcript cleavage activity of RNA polymerase. Proc Natl Acad Sci USA 1995, 92(10):4596-4600.

24. Perederina A, Svetlov V, Vassylyeva MN, Tahirov TH, Yokoyama S, Artsimovitch I, Vassylyev DG: Regulation through the secondary channelstructural framework for ppGpp-DksA synergism during transcription. Cell 2004, 118(3):297-309.

25. Paul BJ, Barker MM, Ross W, Schneider DA, Webb C, Foster JW, Gourse RL: DksA: A critical component of the transcription initiation machinery that potentiates the regulation of rRNA promoters by ppGpp and the initiating NTP. Cell 2004, 118(3):311-322.

26. Blankschien MD, Lee JH, Grace ED, Lennon CW, Halliday JA, Ross W, Gourse RL, Herman C: Super DksAs: substitutions in DksA enhancing its effects on transcription initiation. EMBO J 2009, 28(12):1720-1731.

27. Selby CP, Sancar A: Molecular mechanism of transcription-repair coupling. Science 1993, 260(5104):53-58.

28. Mirkin EV, Mirkin SM: Replication fork stalling at natural impediments. Microbiol Mol Biol Rev 2007, 71(1):13-35.

29. Seigneur M, Bidnenko V, Ehrlich SD, Michel B: RuvAB acts at arrested replication forks. Cell 1998, 95(3):419-430. 
30. Kuzminov A: Collapse and repair of replication forks in Escherichia coli. Mol Microbiol 1995, 16(3):373-384.

31. Stahl MM, Thomason L, Poteete AR, Tarkowski T, Kuzminov A, Stahl FW: Annealing vs. invasion in phage $\lambda$ recombination. Genetics 1997, 147:961-977.

32. Stahl FW, MCMilin KD, Stahl MM, Craseman JM, Lam S: The distribution of crossovers along unreplicated lambda bacteriophage chromosomes. Genetics 1974, 77:395-408.

33. Kang PJ, Craig EA: Identification and characterization of a new Escherichia coli gene that is a dosage-dependent suppressor of a dnaK deletion mutation. J Bacteriol 1990, 172(4):2055-2064.

34. Murphy KC, Campellone KG: Lambda Red-mediated recombinogenic engineering of enterohemorrhagic and enteropathogenic E. coli. BMC Molecular Biology 2003, 4:11.

35. Kolisnychenko V, III GP, Herring CD, Feher T, Posfai J, Blattner FR, Posfai G: Engineering a reduced Escherichia coli genome. Genome Res 2002 12:640-647.

36. Sklar R: Enchancement of nitrosoguanidine mutagenesis by chloramphenicol in Escherichia coli K-12. J Bacteriol 1978, 136(1):460-462

37. Ortenberg R, Gon S, Porat A, Beckwith J: Interactions of glutaredoxins, ribonucleotide reductase, and components of the DNA replication system of Escherichia coli. Proc Natl Acad Sci USA 2004, 101(19):7439-7444.

38. Baba T, Ara T, Hasegawa M, Takai Y, Okumura Y, Baba M, Datsenko KA, Tomita M, Wanner BL, Mori H: Construction of Escherichia coli K-12 inframe, single-gene knockout mutants: the Keio collection. Mol Syst Biol 2006, 2:2006-0008.

39. Kleckner N, Bender J, Gottesman S: Uses of transposons with emphasis on Tn10. Methods Enzymol 1991, 204:139-180.

40. Campbell A: Sensitive mutants of bacteriophage lambda. Virology 1961, 14:22-32.

41. Oppenheim AB, Rattray AJ, Bubunenko M, Thomason LC, Court DL: In vivo recombineering of bacteriophage lambda by PCR fragments and singlestrand oligonucleotides. Virology 2004, 319(2):185-189.

42. Bolivar F, Rodriguez RL, Betlach MC, Boyer HW: Construction and characterization of new cloning vehicles. I. Ampicillin-resistant derivatives of the plasmid pMB9. Gene 1977, 2(2):75-93.

43. Bachmann BJ: Derivations and genotypes of some mutant derivatives of Escherichia coli K-12. In Escherichia coli and Salmonella: cellular and molecular biology. Edited by: Neidhardt FC, III RC, Ingraham JL, Lin ECC, Low KB, Magasanik B, Reznikoff WS, Riley M, Schaechter M, Umbarger HE. Washington, D.C.: ASM Press; 1996:2460-2488.

44. Poteete AR, Fenton AC, Nadkarni A: Chromosomal duplications and cointegrates generated by the bacteriophage lambda Red system in Escherichia coli K-12. BMC Molecular Biology 2004, 5:22

45. Cherepanov PP, Wackernagel W: Gene disruption in Escherichia coli: TcR and $\mathrm{KmR}$ cassettes with the option of Flp-catalyzed excision of the antibiotic-resistance determinant. Gene 1995, 158(1):9-14.

46. Jensen PR, Hammer K: The sequence of spacers between the consensus sequences modulates the strength of prokaryotic promoters. Appl Environ Microbiol 1998, 64(1):82-87.

47. O'Toole GA, Pratt LA, Watnick PI, Newman DK, Weaver VB, Kolter R: Genetic approaches to study of biofilms. Methods Enzymol 1999, 310:91-109.

\section{doi:10.1186/1471-2199-12-12}

Cite this article as: Poteete: Recombination Phenotypes of Escherichia coli greA Mutants. BMC Molecular Biology 2011 12:12.

\section{Submit your next manuscript to BioMed Central and take full advantage of:}

- Convenient online submission

- Thorough peer review

- No space constraints or color figure charges

- Immediate publication on acceptance

- Inclusion in PubMed, CAS, Scopus and Google Scholar

- Research which is freely available for redistribution

Submit your manuscript at www.biomedcentral.com/submit
Biomed Central 